\title{
A clinical perspective of the link between metabolic syndrome and hepatocellular carcinoma
}

This article was published in the following Dove Press journal:

Journal of Hepatocellular Carcinoma

23 February 2015

Number of times this article has been viewed

\section{François Cauchy \\ Jacques Belghiti}

HPB and Liver Transplantation Unit, Hôpital Beaujon, Assistance PubliqueHôpitaux de Paris, Clichy, France
Correspondence: Jacques Belghiti HPB and Liver Transplantation Unit, Hôpital Beaujon, HPB Surgery, Hôpital Beaujon, 100 Boulevard Général Leclerc, 921 I 0 Clichy, France

Tel +33 I 40875895

Fax +33 | 4087 I724

Email jacques.belghiti@bjn.aphp.fr
Abstract: Metabolic syndrome (MS), which is defined as a constellation of clinico-biological features closely related to insulin-resistance has reached epidemic levels in Western Europe and Northern America. Non-alcoholic fatty liver disease (NAFLD) represents the hepatic manifestation of MS. As its incidence parallels that of MS, NAFLD is currently becoming one of the most frequent chronic liver diseases in Western countries. On one hand, MS favors the development of hepatocellular carcinoma (HCC) either through NAFLD liver parenchymal alterations (steatosis; steatohepatitis; fibrosis), or in the absence of significant underlying liver parenchyma changes. In this setting, HCC are often diagnosed incidentally, tend to be larger than in patients developing $\mathrm{HCC}$ on cirrhosis and therefore frequently require major liver resections. On the other hand, MS patients are at increased risk of both liver-related postoperative complications and increased cardiorespiratory events leading to non-negligible mortality rates following liver surgery. These deleterious effects seem to be related to the existence of impaired liver function even in the absence of severe fibrosis but also higher cardiorespiratory sensitivity in a setting of MS/NAFLD. Hence, specific medical and surgical improvements in the perioperative management of these patients are required. These include complete preoperative cardiorespiratory work-up and the wide use of preoperative liver volume modulation. Finally, the long-term prognosis after curative surgery for MS-related HCC does not seem to be worse than for other HCC occurring on classical chronic liver diseases. This is probably related to less aggressive tumor behavior with lower micro vascular invasion and decreased rates of poorly differentiated lesions. In this setting, several medical therapies including metformin could be of value in the prevention of both occurrence and recurrence of HCC.

Keywords: metabolic syndrome, NAFLD, NASH, neoplasia, hepatocellular carcinoma, hepatectomy, complications, morbidity

\section{Introduction}

The prevalence of metabolic syndrome (MS) has reached epidemic levels in Western Europe and Northern America, where it is reported to be as high as $25 \% .{ }^{1} \mathrm{MS}$ is defined as a constellation of clinico-biological features closely related to insulin-resistance and includes dyslipidemia, hypertension, glucose intolerance and central obesity. ${ }^{1}$ Non-alcoholic fatty liver disease (NAFLD) accounts for the hepatic manifestation of MS. NAFLD ranges from simple steatosis to steatohepatitis and may lead to fibrosis and endstage liver disease. ${ }^{2}$ As its incidence parallels that of MS, NAFLD has become one of the most frequent chronic liver diseases in Western countries. ${ }^{3}$ Also, it has been suggested that both MS and NAFLD could directly or indirectly promote the development of primary liver malignancies. ${ }^{4-7}$ Hence, it is likely that more and more of these patients will be referred in hepatobiliary and liver transplant units in upcoming years. ${ }^{8}$ 
Despite increasing concerns regarding the growing incidence of MS/NAFLD-related liver malignancy, the specific impact of both MS and NAFLD on the postoperative course of patients undergoing liver surgery has long been neglected. Indeed, it is only recently that evidence suggesting an underestimated risk regarding postoperative morbidity and mortality in MS patients undergoing liver surgery has been released. ${ }^{8-13}$ In that sense, clinicians should be fully aware that the existence of MS and NAFLD may have a negative impact on the postoperative course of their patients in order to optimize perioperative management and prevent avoidable morbidity/mortality.

The present review aims to provide comprehensive insights regarding the current standards and issues in the diagnosis of both MS and NAFLD in order to clarify their respective impact on tumor progression as well as their influence on the postoperative outcome. Finally, we will discuss the measures which should be undertaken in upcoming years in order to improve the results of surgery.

\section{Issues regarding the use of current definitions in liver surgery Metabolic syndrome}

The definition of MS has evolved during the past decade. Current consensual criteria for its diagnosis include central obesity, hypertension, dyslipidemia (with either increased triglycerides level or decreased high density lipoprotein cholesterol level), and glucose intolerance. ${ }^{1}$ The presence of at least three out of five of the abovementioned criteria are required to confirm the existence of MS. ${ }^{1}$ Yet, liver histological manifestations and influence on surgical outcomes after liver surgery may occur in patients presenting with individual components of MS. Indeed, fatty liver disease may also occur in patients with isolated diabetes mellitus (DM) ${ }^{14}$ hypertriglyceridemia, ${ }^{15}$ and obesity. ${ }^{16,17}$ Likewise, higher perioperative morbidity or mortality rates after liver resection have been reported in patients with only $\mathrm{DM},{ }^{18,19}$ or who are overweight/obese, ${ }^{20,21}$ or the association of several of these disorders. ${ }^{13,22}$

Interestingly, most surgical studies, because of their retrospective nature, do not gather all these consensual criteria but rather use substitutes for convenience. Such substitutes may lead to a certain degree of confusion. For example, it is frequently assumed that patients receiving statin or fenofibrate medication have dyslipidemia ${ }^{8,11}$ and that patients receiving antihypertensive therapy have hypertension. However, some of these medications may be used for primary cardiovascular prevention or renal protection in the absence of MS disorders.
In the same way, central obesity and visceral adiposity are often assessed using the body mass index. In this setting various cut-off values have been proposed. ${ }^{8,12,13}$ Yet, BMI does not distinguish between central obesity, which is included in MS, from peripheral obesity. In that sense, circumference of waist appears to be more reliable and should be preferentially used. ${ }^{23,24}$ Finally, the terms hyperglycemia and insulin-resistance are often used indiscriminately, whereas some authors suggest that they should not. ${ }^{1,25}$ Hence, the presence of insulin-resistance should be routinely assessed using the homeostasis model assessment of insulin resistance (HOMA-IR $)^{25}$ whenever hyperglycemia is found.

\section{NAFLD}

NAFLD has become one of the most frequent forms of chronic liver disease in Western countries. ${ }^{5,6}$ The presence of NAFLD is defined by a fatty infiltration exceeding $5 \%$ of the liver parenchyma at histology in the absence of previous or ongoing significant alcohol consumption. ${ }^{26}$ However, one should nevertheless bear in mind that a significant proportion of MS patients with fatty liver changes also have a history of daily alcohol consumption above 20-30 g per day and strict definition of NAFLD probably underestimates its true prevalence among MS patients. Although NAFLD is considered the hepatic manifestation of MS, other conditions including chronic hepatitis $\mathrm{B}$ and $\mathrm{C}$ infection, ${ }^{27,28}$ irinotecan-based chemotherapy ${ }^{29,30}$ and several other medications including methotrexate, tamoxifen or amiodarone $\mathrm{e}^{31,32}$ may also lead to fatty liver disease and should be meticulously ruled out. NAFLD, which encompasses a wide spectrum of diseases ranging from simple steatosis to non-alcoholic steatohepatitis (NASH), ${ }^{26}$ can progress to cirrhosis and may lead to end-stage liver disease. ${ }^{5,6}$ Histological analysis remains the gold standard for the assessment of NAFLD and should be performed by a trained pathologist. ${ }^{33}$ Several histological scores might be useful for the diagnosis of NAFLD. The most frequently used score is the non-alcoholic liver disease activity score (NAS) proposed by Kleiner et al, ${ }^{26}$ which is a semiquantitative, histology-based score system including three parameters, namely steatosis (on a scale of 0 to 3 ), lobular inflammation and hepatocellular ballooning (on a scale of 0 to 2 each). More recently, Bedossa et al published a histopathological algorithm and scoring system for the evaluation of liver lesions in morbidly obese patients. ${ }^{34} \mathrm{In}$ this setting, the authors observed that this so called SAF score based on steatosis (S), activity (A) of the disease (ballooning and lobular inflammation) and fibrosis (F) grade in the underlying liver allowed decreasing interobserver varia- 
tions among pathologists and was likely to be implemented in routine pathology practice. ${ }^{35}$

\section{$\mathrm{NASH}$}

NASH is considered the result of long-lasting inflammation following fatty liver infiltration. It is characterized by several histological alterations, including steatosis, lobular inflammation, and ballooning and may also be associated with fibrosis. Even though the diagnosis of NASH was initially suggested for NAS values of 4 or $5,{ }^{26}$ there is an ongoing debate regarding the accuracy of NAS in assessing NASH. Interestingly, Brunt et al have emphasized that the diagnosis of NASH based on the evaluation of patterns as well as individual lesions on liver biopsies did not always correlate with threshold values of the semiquantitative NAS. ${ }^{33}$ Thus, rather than being based on the NAS value alone, the differentiation between NASH and no-NASH should better take into account the pathologist report. ${ }^{33}$

\section{Identification of NASH in patients with MS/NAFLD}

Since the increasing incidence of both MS and NAFLD in Western populations put a great amount of patients at risk of developing NASH, any large-scale screening policy aimed to obtain histological diagnosis of NAFLD is unlikely to be reasonably performed. Furthermore, the accuracy of histology in identifying NASH is suboptimal as both interobserver variations $^{36}$ and discrepancies from one sample to the other within the same parenchyma may occur. ${ }^{37}$ In order to increase cost-effectiveness and accuracy of diagnosis, and also to avoid the intrinsic invasiveness of biopsy, there has been significant interest in identifying non-invasive methods of predicting liver histology in patients with suspected NASH. Hence, numerous biological (ALT/AST ratio; FIB-4; analysis of organic compounds in breath), ${ }^{38}$ and imaging techniques (magnetic resonance imaging [MRI] for quantification of liver steatosis ${ }^{39}$ or magnetic resonance spectroscopy) have been proposed for the detection of underlying parenchymal changes among patients with MS, but none has yet become the gold standard. In particular, although MRI has shown high accuracy in detecting steatosis, its effectiveness in evaluating (and possibly ruling out) fibrosis remains questionable in the presence of fat. ${ }^{40}$

\section{MS/NAFLD influence on primary liver carcinogenesis}

Two recent series have shown that MS itself was associated with an increased risk of developing HCC. ${ }^{3,41}$ In this setting, $\mathrm{HCC}$ incidence in patients with MS has been reported to be
2-4 fold higher than in the general population. ${ }^{7}$ How MS acts to promote carcinogenesis remains to be fully elucidated. In this setting, liver tumorigenesis might be indeed complex, involving potential direct pro-tumoral actions of insulin-resistance ${ }^{42}$ and obesity ${ }^{43}$ but also indirect effects related to the development of underlying NAFLD. ${ }^{5,6}$ On one hand, direct oncologic effects may be the consequence of low-grade, chronic systemic inflammation, implying a serum increase of inflammatory cytokines such as TNF- $\alpha$ and IL- $6^{5}$ and a decrease in anti-inflammatory ones including adipocytokines $^{44}$ potentially leading to loss of tumor suppression genes and deregulation of several signaling pathways such as the AMPK/mammalian target of rapamycin (mTOR) pathway. ${ }^{45}$ This mechanism likely explains the absence of severe underlying fibrosis in as much as $30 \%-60 \%$ of the patients with MS-related $\mathrm{HCC}^{7,8,22,46}$ including almost $20 \%$ of patients with a strictly normal underlying liver parenchyma. On the other hand, although not always present, NASH-related cirrhosis may be possibly considered a precancerous lesion, as it is associated with a yearly incidence of $\mathrm{HCC}$ as high as $2.6 \%{ }^{5}$ leading to a cumulative five-year incidence ranging from $7.6 \%{ }^{47}$ to $11 \%{ }^{48}$ In the event of NASH-related cirrhosis, both the presence and pattern of hepatic iron deposition ${ }^{49,50}$ as well as any regular alcohol consumption ${ }^{6}$ have been incriminated to further accentuate parenchymal changes thus promoting liver carcinogenesis.

Finally, viral infection may also play an indirect role in tumor development in patients with MS. In particular, the specific subset of patients with chronic hepatitis $\mathrm{C}$ virus (HCV) infection developing an HCC is worth mentioning. Several authors have emphasized that chronic HCV infection was associated with fatty infiltration of the liver parenchyma in $50 \%-70 \%$ of the cases, including massive steatosis and NASH. ${ }^{27,28,51,52}$ A significant number of the latter display the so-called "viral steatosis" as a consequence of virus interference with fat metabolism (in the absence of pre-existing metabolic disorders). Thus, in this setting, steatosis itself could be responsible for the occurrence of secondary insulinresistance and systemic inflammation. Even though the viral steatosis has been shown to regress after viral eradication, ${ }^{53}$ its existence has been incriminated in recurrence of $\mathrm{HCV}$ related $\mathrm{HCC}^{54}$ after curative surgery. However, since steatosis and lobular inflammation may be found in HCV infection regardless of MS/NAFLD, the supposed association between $\mathrm{HCC}, \mathrm{HCV}$, and NAFLD could be more a statistical artifact than a real oncogenetic mechanism. Taken together, the supposed pathway from viral infection to viral steatosis and $\mathrm{HCC}$, as well as the possible mechanisms finally leading 
to HCC development (fibrosis, inflammation, or induced insulin-resistance), still remain to be assessed.

\section{MS/NAFLD impact on outcome of liver surgery}

The impact of individual components of MS and liver steatosis on the postoperative course following liver resection has been extensively investigated. ${ }^{18,55-59}$ Accordingly, it has been established that liver surgery provided poorer results in patients affected by diabetes ${ }^{18}$ or obesity ${ }^{55,56}$ than in otherwise healthy patients. Similarly, several studies have highlighted that steatosis per se was a risk factor for postoperative complications after major hepatectomy. ${ }^{57-60}$ In experimental models, liver fatty infiltration such as mild or severe steatosis has been found to be associated with lower regenerative ability following portal vein occlusion, elevated sensitivity to ischemia-reperfusion injury, and higher hepatocellular injury after partial liver resection. ${ }^{61}$ Nevertheless, it is only recently that surgeons have focused on the results of liver resection and transplantation, in the specific subset of patients with MS or NASH.

\section{Liver resection}

Up to now, only six studies have analyzed the early outcome of patients undergoing liver resection in a setting of MS/NASH. ${ }^{8-13}$ Of these, three aimed at assessing the influence of MS on outcome, ${ }^{8,12,13}$ whereas the remaining three aimed at evaluating the impact of histological modifications, including NAFLD and NASH. ${ }^{9-11}$ Overall, mortality after liver resection varied from $3 \%$ up to $30 \%$, and was related to the primarily studied parameter ie, MS, NAFLD or NASH. In this setting, it has been recently suggested that MS patients with a NAS $>2^{8}$ or those with an histological diagnosis of $\mathrm{NASH}^{11}$ had a 2.7-fold greater risk of experiencing liverrelated but also cardiorespiratory complications than those with normal underlying parenchyma. Hence, it seems that steatohepatitis rather than simple steatosis was a risk factor for postoperative complications. ${ }^{11}$ Even if these recent findings may appear in opposition with previously published results maintaining a negative impact of steatosis on outcome, ${ }^{58-60}$ it is likely that the poor assessment of inflammatory changes in the underlying steatotic parenchyma may have biased older series. However, the progressive increasing degree of parenchymal change, damage and inflammation from steatosis to steatohepatitis is nowadays considered as a continuum, which progressively and proportionally increases overall postoperative morbidity/mortality.

Intuitively, not only the "quality" but also the "quantity" of liver remnant should be considered. In fact, it has been recently suggested that NASH was independently associated with both higher postoperative liver insufficiency and mortality following right hepatectomy (including extended right hepatectomy), ${ }^{13}$ and trisectionectomy, ${ }^{10}$ although a safe amount of liver parenchyma was left in place. This result clearly emphasizes the inferior tolerance to extended resection of fatty and inflammatory livers. This feature may be of particular importance in the case of HCC developing in a MS/NAFLD context, where large lesions often require major resections..$^{8,22}$

Considering cardiovascular morbidity/mortality, it has been shown that NASH was an independent risk-factor for the development of coronary artery disease and calcifications regardless of the degree of visceral adiposity, ${ }^{62,63}$ thus leading to higher incidence of cardiorespiratory events following liver resection. Possibly, the recently described hemorheological alterations occurring in MS patients, including increased erythrocyte aggregation, ${ }^{64,65}$ may also play a role in ischemic cardiac events. Likewise, even though not documented, potential changes in coagulation in MS patients could influence the postoperative course of these patients. This is particularly true following major resection after which the normal coagulation profile is largely modified but also considering the pre-existing pro-thrombotic state in the absence of underlying cirrhosis leading to increased risk of pulmonary embolism. ${ }^{66}$

\section{Liver transplantation}

NASH can progress to cirrhosis ${ }^{2,4}$ and may lead to end-stage liver disease requiring liver transplantation (LT). During the last decade, the rate of LT performed for NASH-related end-stage liver disease has dramatically increased from about $3 \%$ in the early 2000 s up to $19 \%$ in $2011 .{ }^{2}$ Currently, NASH is the third most common cause of LT in the US and is about to become the most common within the next two decades in Western countries. ${ }^{67}$

LT in NASH patients has peculiar aspects. Compared with other patients undergoing LT, recipients with NASH tend to be older ${ }^{68}$ and obviously have a higher frequency of metabolic disorders. ${ }^{62}$ In this setting, procedures significantly last longer and are associated with higher blood loss and longer post-transplantation hospital stays. ${ }^{62}$ Accordingly, 30-day mortality after LT in patients with NASH tends to be higher than that for other indications. ${ }^{69}$ Several studies have reported increased liver related morbidity rates in NASH patients, such as acute rejection rates ${ }^{68}$ but also extra-hepatic complications, including sepsis and renal dysfunction. ${ }^{70}$ Similarly to patients undergoing hepatectomy, NASH patients 
undergoing LT also have a higher likelihood of developing cardiovascular complications. ${ }^{62,68,70}$ These events, which mainly occur within the first year after LT, have been reported to be responsible for as high as $50 \%$ of the total mortality following LT. ${ }^{62}$ The relationship between MS/NASH and cardiovascular morbidity seems more complex than a generic multi-organ vascular disorder due to MS, as suggested by the significantly higher occurrence of cardiovascular events associated with MS whenever NASH is present. ${ }^{71}$ In fact, similarly to what has been observed after liver resection (LR), NASH is nowadays thought to put patients at an even higher risk of cardiovascular complications, regardless of comorbidities and patient-specific cardiac risk. ${ }^{62}$ Here again, it is likely that the degree of inflammation in the underlying liver represents a key factor in the occurrence of increased cardiovascular sensitivity.

Long-term results of LT for NASH are encouraging. One-, 3- and 5-year survivals after LT for NASH range from $84 \%-87.6 \%, 75 \%-82.2 \%$, and $70 \%-76.7 \%$, respectively, and are at least similar to those observed for LT for other traditional indications. ${ }^{2,62,68,69,72}$ More interestingly, LT for $\mathrm{HCC}$ developed in patients with NASH seems to provide an excellent long-term outcome with higher survivals compared with patients transplanted for $\mathrm{HCV}$-related HCC. ${ }^{73}$ These observations could be the result of less aggressive tumors in NASH patients with lower vascular invasion and decreased rates of poorly differentiated lesions. ${ }^{74}$

LT in patients with NASH-related cirrhosis presents peculiar issues, including cirrhosis. Recurrent disease after LT for NASH-related cirrhosis has been reported to occur in as high as $34 \%$ of recipients. ${ }^{69,75}$ There is little information detailing the occurrence and histological evolution of NAFLD recurrence after LT, and the long-term natural history of NAFLD recurrence itself is unclear. ${ }^{76}$ Nevertheless, in these patients, recurrence is often associated with the presence of MS or its individual components. ${ }^{75}$ Accordingly, recurrence should be further evaluated in larger studies, with special emphasis on management of MS and secondary prevention strategies. $^{75}$

\section{Which improvements should be undertaken in upcoming years?}

Both MS and NAFLD/NASH adversely affect short and long-term results of liver surgery. Considering that the rate of patients presenting with such conditions will keep on increasing in upcoming years, it appears crucial that specific measures should be undertaken in order to improve those unsatisfactory results. Above all, the inferior tolerance to extended resection of fatty and inflammatory livers (as a consequence of lower regenerative ability), requires specific focus during the preoperative planning of surgical strategy whenever a major resection is needed. Unfortunately, the culture of considering just MS or steatosis (even without liver biopsy confirmation) a potential risk factor for major surgery has not entered clinical practice even in specialized environments. Addressing this issue, our group has recently shown that MS patients operated on for HCC less frequently underwent preoperative portal vein embolization (PVE) when they displayed a NAS $>2$ without severe fibrosis compared to those with severe underlying fibrosis, suggesting that these latter patients would probably benefit from a better anticipation of their operative risk, especially in the case of planned major LR. ${ }^{8}$

In general, preventing measures to reduce MS/NAFLDrelated morbidity/mortality should include: i) better characterization of the underlying parenchyma using invasive or non-invasive means knowing that patients with inflammatory fatty liver even without severe fibrosis are at similar operative risk as those with severe underlying fibrosis; ii) MS targeted perioperative management including complete perioperative cardiorespiratory work-up and monitoring as well as continuous postoperative blood glucose control;77,78 and iii) specific, "NAFLD-tailored" perioperative surgical care, such as parenchymal-sparing resections, wide use of liver volume modulation techniques, including portal vein embolization and portal vein ligation, but also targeted medical therapies developed in order to improve the tolerance to LR. Concerning this latter issue, a recent experimental study has highlighted the benefits of omega- 3 acids in reducing severe steatosis in a preoperative setting leading to improved liver regeneration and functional recovery following partial hepatectomy. ${ }^{79}$ These encouraging preliminary results yet require confirmation in a clinical setting but may already be considered a promising future field of research.

Concerning the relationship between MS/NAFLD and neoplastic disease, several strategies should be developed in order to prevent both occurrence and recurrence of primary liver cancer in MS/NASH patients. Even though it is generally recommended that overweight and obese patients with NAFLD lose $7 \%-10 \%$ of their body weight by dietary modification and exercise over the course of 6-12 months, the paucity of data makes it difficult to make evidence-based recommendations about dietary modification and exercise to treat NAFLD and NASH. ${ }^{80}$ In fact, medical research has mainly focused on reducing NASH in MS patients using 
medical therapies. Several randomized controlled trials have shown significant downstaging of NASH following the administration of specific medications, including vitamin $\mathrm{E}$ and pioglitazone. ${ }^{81-83}$ More recently, retrospective studies have shown that the use of biguanides, such as metformin, was associated with a systematic $\mathrm{HCC}$ risk reduction among diabetic patients while this risk was not decreased using several other antidiabetic therapies. ${ }^{84,85}$ While the precise antitumoral mechanisms of metformin in a setting of HCC still remain to be fully elucidated, there is growing evidence that LKB1-dependent and AMPK-dependent suppression of the mTOR pathway is possibly the most potent antineoplastic effect of metformin. ${ }^{86,87}$ Indeed, mTOR inhibition disturbs protein synthesis and, thereby, tumor cell proliferation. ${ }^{87}$ Hence, several therapies aiming at inhibiting the mTOR pathway have recently proved efficient in patients with various malignancies including breast cancer, ${ }^{88}$ renal cell carcinoma ${ }^{89}$ and pancreatic endocrine tumour. ${ }^{90}$ Experimentally, metformin has recently shown promising results in the prevention of HCC development ${ }^{91}$ but also in the limitation of tumor growth in rodents. ${ }^{92-95}$ Altogether, in a context of MS/NAFLD related HCC, metformin would theoretically represent an ideal preventing therapy limiting the influence of type II diabetes in the occurrence of HCC but also providing inherent antitumoral properties. Nevertheless, despite the encouraging results of all these medications and the possible future development of others even more effective, it should be kept in mind that none of them have currently been tested in a surgical context. In fact, the prolonged time interval required by medications to obtain relevant effects on liver parenchyma possibly reducing morbidity, definitely questions its applicability in a surgical environment prior to surgery. This consideration gains interest if one considers that the great majority of patients undergoing major liver surgery (LR and LT) present with cancer or end-stage liver disease, needing prompt management. Obviously, any medical/preventing strategy should ideally require a large-scale evaluation in a surgical setting.

\section{Conclusion}

Both the pro-oncogenic effect on the underlying liver and the rising incidence of MS/NASH imply that an increased number of patients with such conditions referred to hepatobiliary units is to be expected. The higher operative risk observed in these patients can be partially explained by both underestimated liver-related risk but also high perioperative cardiovascular and respiratory susceptibility. These unsatisfactory postoperative results will require targeted perioperative management. Such actions are justified by the observed favorable long-term outcomes.

\section{Disclosure}

The authors report no conflict of interest in this work.

\section{References}

1. Eckel RH, Alberti KG, Grundy SM, Zimmet PZ. The metabolic syndrome. Lancet. 2010;375(9710):181-183.

2. Agopian VG, Kaldas FM, Hong JC, et al. Liver transplantation for nonalcoholic steatohepatitis: the new epidemic. Ann Surg. 2012;256(4):624-633.

3. Fierbinteanu-Braticevici C, Negreanu L, Tarantino G. Is fatty liver always benign and should not consequently be treated? J Physiol Pharmacol. 2013;64(1):3-9.

4. Welzel TM, Graubard BI, Zeuzem S, El-Serag HB, Davila JA, McGlynn KA. Metabolic syndrome increases the risk of primary liver cancer in the United States: a study in the SEER-Medicare database. Hepatology. 2011;54(2):463-471.

5. Starley BQ, Calcagno CJ, Harrison SA. Nonalcoholic fatty liver disease and hepatocellular carcinoma: a weighty connection. Hepatology. 2010;51(5):1820-1832.

6. Ascha MS, Hanouneh IA, Lopez R, Tamimi TA, Feldstein AF, Zein NN. The incidence and risk factors of hepatocellular carcinoma in patients with nonalcoholic steatohepatitis. Hepatology. 2010;51(6):1972-1978.

7. Turati F, Talamini R, Pelucchi C, et al. Metabolic syndrome and hepatocellular carcinoma risk. Br J Cancer. 2013;108(1):222-228.

8. Cauchy F, Zalinski S, Dokmak S, et al. Surgical treatment of hepatocellular carcinoma associated with the metabolic syndrome. Br J Surg. 2013;100(1):113-121.

9. Wakai T, Shirai Y, Sakata J, Korita PV, Ajioka Y, Hatakeyama K. Surgical outcomes for hepatocellular carcinoma in nonalcoholic fatty liver disease. J Gastrointest Surg. 2011;15(8):1450-1458.

10. Neal CP, Mann CD, Pointen E, et al. Influence of hepatic parenchymal histology on outcome following right hepatic trisectionectomy. J Gastrointest Surg. 2012;16(11):2064-2073.

11. Reddy SK, Marsh JW, Varley PR, et al. Underlying steatohepatitis, but not simple hepatic steatosis, increases morbidity after liver resection: A case-control study. Hepatology. 2012;56(6):2221-2230.

12. Bhayani NH, Hyder O, Frederick W, et al. Effect of metabolic syndrome on perioperative outcomes after liver surgery: A National Surgical Quality Improvement Program (NSQIP) analysis. Surgery. 2012;152(2): 218-226.

13. Zarzavadjian Le Bian A, Costi R, Constantinides V, Smadja C. Metabolic disorders, non-alcoholic fatty liver disease and major liver resection: an underestimated perioperative risk. J Gastrointest Surg. 2012;16(12):2247-2255.

14. El-Serag HB, Tran T, Everhart JE. Diabetes increases the risk of chronic liver disease and hepatocellular carcinoma. Gastroenterology. 2004;126(2):460-468.

15. Fiatarone JR, Coverdale SA, Batey RG, Farrell GC. Non-alcoholic steatohepatitis: impaired antipyrine metabolism and hypertriglyceridaemia may be clues to its pathogenesis. $J$ Gastroenterol Hepato. 1991;6(6):585-590.

16. Ratziu V, Giral P, Charlotte F, et al. Liver fibrosis in overweight patients. Gastroenterology. 2000;118(6):1117-1123.

17. Gholam PM, Flancbaum L, Machan JT, Charney DA, Kotler DP. Nonalcoholic fatty liver disease in severely obese subjects. Am J Gastroenterol. 2007;102(2):399-408.

18. Huo TI, Lui WY, Huang YH, et al. Diabetes mellitus is a risk factor for hepatic decompensation in patients with hepatocellular carcinoma undergoing resection: a longitudinal study. Am J Gastroenterol. 2003;98(10):2293-2298. 
19. Slankamenac K, Breitenstein S, Held U, Beck-Schimmer B, Puhan MA, Clavien PA. Development and validation of a prediction score for postoperative acute renal failure following liver resection. Ann Surg. 2009;250(5):720-728.

20. Pathak S, Tang JM, Terlizzo M, Poston GJ, Malik HZ. Hepatic steatosis, body mass index and long term outcome in patients undergoing hepatectomy for colorectal liver metastases. Eur J Surg Oncol. 2010;36(1):52-57.

21. MathurAK, GhaferiAA, Sell K, Sonnenday CJ, Englesbe MJ, WellingTH. Influence of body mass index on complications and oncologic outcomes following hepatectomy for malignancy. J Gastrointest Surg. 2010;14(5):849-857.

22. Paradis V, Zalinski S, Chelbi E, et al. Hepatocellular carcinomas in patients with metabolic syndrome often develop without significant liver fibrosis: a pathological analysis. Hepatology. 2009;49(3):851-859.

23. Janssen I, Katzmarzyk PT, Ross R. Body mass index, waist circumference, and health risk: evidence in support of current National Institutes of Health guidelines. Arch Intern Med. 2002;162(18):2074-2079.

24. Janssen I, Katzmarzyk PT, Ross R. Waist circumference and not body mass index explains obesity-related health risk. Am J Clin Nutr. 2004;79(3):379-384.

25. Bonora E, Targher G, Alberiche M, et al. Homeostasis model assessment closely mirrors the glucose clamp technique in the assessment of insulin sensitivity: studies in subjects with various degrees of glucose tolerance and insulin sensitivity. Diabetes Care. 2000;23(1):57-63.

26. Kleiner DE, Brunt EM, Van Natta M, et al; Nonalcoholic Steatohepatitis Clinical Research Network. Design and validation of a histological scoring system for nonalcoholic fatty liver disease. Hepatology. 2005;41(6):1313-1321.

27. Bedossa P, Moucari R, Chelbi E, et al. Evidence for a role of nonalcoholic steatohepatitis in hepatitis C: a prospective study. Hepatology 2007;46(2):380-387.

28. Moucari R, Asselah T, Cazals-Hatem D, et al. Insulin resistance in chronic hepatitis $\mathrm{C}$ : association with genotypes 1 and 4, serum HCV RNA level, and liver fibrosis. Gastroenterology. 2008;134(2):416-423.

29. Fernandez FG, Ritter J, Goodwin JW, Linehan DC, Hawkins WG, Strasberg SM. Effect of steatohepatitis associated with irinotecan or oxaliplatin pretreatment on resectability of hepatic colorectal metastases. J Am Coll Surg. 2005;200(6):845-853.

30. Vauthey JN, Pawlik TM, Ribero D, et al. Chemotherapy regimen predicts steatohepatitis and an increase in 90-day mortality after surgery for hepatic colorectal metastases. J Clin Oncol. 2006;24(13):2065-2072.

31. Angulo P. Nonalcoholic fatty liver disease. $N$ Engl J Med. 2002; 346(16):1221-1231.

32. Vuppalanchi R, Chalasani N. Nonalcoholic fatty liver disease and nonalcoholic steatohepatitis: Selected practical issues in their evaluation and management. Hepatology. 2009;49(1):306-317.

33. Brunt EM, Kleiner DE, Wilson LA, Belt P, Neuschwander-Tetri BA; NASH Clinical Research Network (CRN). Nonalcoholic fatty liver disease (NAFLD) activity score and the histopathologic diagnosis in NAFLD: distinct clinicopathologic meanings. Hepatology. 2011;53(3): 810-820.

34. Bedossa P, Poitou C, Veyrie N, et al. Histopathological algorithm and scoring system for evaluation of liver lesions in morbidly obese patients. Hepatology. 2012;56(5):1751-1759.

35. Bedossa P; FLIP Pathology Consortium. Utility and appropriateness of the fatty liver inhibition of progression (FLIP) algorithm and steatosis, activity, and fibrosis (SAF) score in the evaluation of biopsies of nonalcoholic fatty liver disease. Hepatology. 2014;60(2):565-575.

36. Merriman RB, Ferrell LD, Patti MG, et al. Correlation of paired liver biopsies in morbidly obese patients with suspected nonalcoholic fatty liver disease. Hepatology. 2006;44(4):874-880.

37. Ratziu V, Charlotte F, Heurtier A, et al; LIDO Study Group. Sampling variability of liver biopsy in nonalcoholic fatty liver disease. Gastroenterology. 2005;128(7):1898-1906.
38. McPherson S, Stewart SF, Henderson E, Burt AD, Day CP. Simple non-invasive fibrosis scoring systems can reliably exclude advanced fibrosis in patients with non-alcoholic fatty liver disease. Gut. 2010;59(9):1265-1269.

39. Raptis DA, Fischer MA, Graf R, et al. MRI: the new reference standard in quantifying hepatic steatosis? Gut. 2012;61(1):117-127.

40. Bülow R, Mensel B, Meffert P, Hernando D, Evert M, Kühn JP. Diffusion-weighted magnetic resonance imaging for staging liver fibrosis is less reliable in the presence of fat and iron. Eur Radiol. 2013;23(5):1281-1287.

41. Jinjuvadia R, Patel S, Liangpunsakul S. The association between metabolic syndrome and hepatocellular carcinoma: systemic review and meta-analysis. J Clin Gastroenterol. 2014;48(2):172-177.

42. Arase Y, Kobayashi M, Suzuki F, et al. Effect of type 2 diabetes on risk for malignancies includes hepatocellular carcinoma in chronic hepatitis C. Hepatology. 2013;57(3):964-973.

43. Calle EE, Rodriguez C, Walker-Thurmond K, Thun MJ. Overweight, obesity, and mortality from cancer in a prospectively studied cohort of US adults. $N$ Engl J Med. 2003;348(17):1625-1638.

44. Michelotti GA, Machado MV, Diehl AM. NAFLD, NASH and liver cancer. Nat Rev Gastroenterol Hepatol. 2013;10(11):656-665.

45. Saxena NK, Fu PP, Nagalingam A, et al. Adiponectin modulates C-jun N-terminal kinase and mammalian target of rapamycin and inhibits hepatocellular carcinoma. Gastroenterology. 2010;139(5):1762-1773.

46. Ertle J, Dechêne A, Sowa JP, et al. Non-alcoholic fatty liver disease progresses to hepatocellular carcinoma in the absence of apparent cirrhosis. Int J Cancer. 2011;128(10):2436-2443.

47. Hashimoto E, Yatsuji S, Tobari M, et al. Hepatocellular carcinoma in patients with nonalcoholic steatohepatitis. $J$ Gastroenterol. 2009;44(Suppl 19):89-95.

48. Yatsuji S, Hashimoto E, Tobari M, Taniai M, Tokushige K, Shiratori K. Clinical features and outcomes of cirrhosis due to non-alcoholic steatohepatitis compared with cirrhosis caused by chronic hepatitis C. J Gastroenterol Hepatol. 2009;24(2):248-254.

49. Sorrentino P, D’Angelo S, Ferbo U, Micheli P, Bracigliano A, Vecchione R. Liver iron excess in patients with hepatocellular carcinoma developed on non-alcoholic steato-hepatitis. J Hepatol. 2009;50(2):351-357.

50. Valenti L, Fracanzani AL, Bugianesi E, et al. HFE genotype, parenchymal iron accumulation, and liver fibrosis in patients with nonalcoholic fatty liver disease. Gastroenterology. 2010;138(3):905-912.

51. Asselah T, Rubbia-Brandt L, Marcellin P, Negro F. Steatosis in chronic hepatitis C: why does it really matter? Gut. 2006;55(1):123-130.

52. Serfaty L, Andreani T, Giral P, Carbonell N, Chazouillères O, Poupon R. Hepatitis C virus induced hypobetalipoproteinemia: a possible mechanism for steatosis in chronic hepatitis C. J Hepatol. 2001;34(3):428-434.

53. Serfaty L, Poujol-Robert A, Carbonell N, Chazouillères O, Poupon RE, Poupon R. Effect of the interaction between steatosis and alcohol intake on liver fibrosis progression in chronic hepatitis C. Am J Gastroenterol. 2002;97(7):1807-1812 .

54. Takuma Y, Nouso K, Makino Y, et al. Hepatic steatosis correlates with the postoperative recurrence of hepatitis C virus-associated hepatocellular carcinoma. Liver Int. 2007;27(5):620-626.

55. Viganò L, Capussotti L, De Rosa G, De Saussure WO, Mentha G, Rubbia-Brandt L. Liver resection for colorectal metastases after chemotherapy: impact of chemotherapy-related liver injuries, pathological tumor response, and micrometastases on long-term survival. Ann Surg. 2013;258(5):731-742.

56. Balzan S, Nagarajan G, Farges O, et al. Safety of liver resections in obese and overweight patients. World J Surg. 2010;34(12):2960-2968.

57. Cucchetti A, Cescon M, Ercolani G, Di Gioia P, Peri E, Pinna AD. Safety of hepatic resection in overweight and obese patients with cirrhosis. Br J Surg. 2011;98(8):1147-1154.

58. McCormack L, Petrowsky H, Jochum W, Furrer K, Clavien PA. Hepatic steatosis is a risk factor for postoperative complications after major hepatectomy: a matched case-control study. Ann Surg. 2007;245(6):923-930. 
59. Veteläinen R, van Vliet A, Gouma DJ, van Gulik TM. Steatosis as a risk factor in liver surgery. Ann Surg. 2007;245(1):20-30.

60. de Meijer VE, Kalish BT, Puder M, Ijzermans JN. Systematic review and meta-analysis of steatosis as a risk factor in major hepatic resection. Br J Surg. 2010;97(9):1331-1339.

61. Veteläinen R, van Vliet AK, van Gulik TM. Severe steatosis increases hepatocellular injury and impairs liver regeneration in a rat model of partial hepatectomy. Ann Surg. 2007;245(1):44-50.

62. Vanwagner LB, Bhave M, Te HS, Feinglass J, Alvarez L, Rinella ME. Patients transplanted for nonalcoholic steatohepatitis are at increased risk for postoperative cardiovascular events. Hepatology. 2012;56(5):1741-1750.

63. Wong VW, Wong GL, Yip GW, et al. Coronary artery disease and cardiovascular outcomes in patients with non-alcoholic fatty liver disease. Gut. 2011;60(12):1721-1727.

64. Vayá A, Hernández-Mijares A, Bonet E, et al. Association between hemorheological alterations and metabolic syndrome. Clin Hemorheol Microcirc. 2011;49(1-4):493-503.

65. Gyawali P, Richards RS, Hughes DL, Tinley P. Erythrocyte aggregation and metabolic syndrome. Clin Hemorheol Microcirc. 2014;57(1):73-83.

66. Melloul E, Dondéro F, Vilgrain V, Raptis DA, Paugam-Burtz C, Belghiti J. Pulmonary embolism after elective liver resection: a prospective analysis of risk factors. J Hepatol. 2012;57(6):1268-1275.

67. Charlton MR, Burns JM, Pedersen RA, Watt KD, Heimbach JK, Dierkhising RA. Frequency and outcomes of liver transplantation for nonalcoholic steatohepatitis in the United States. Gastroenterology. 2011;141(4):1249-1253.

68. Bhagat V, Mindikoglu AL, Nudo CG, Schiff ER, Tzakis A, Regev A. Outcomes of liver transplantation in patients with cirrhosis due to nonalcoholic steatohepatitis versus patients with cirrhosis due to alcoholic liver disease. Liver Transpl. 2009;15(12):1814-1820.

69. Malik SM, deVera ME, Fontes P, Shaikh O, Ahmad J. Outcome after liver transplantation for NASH cirrhosis. Am J Transplant. 2009;9:782-793.

70. Houlihan DD, Armstrong MJ, Davidov Y, et al. Renal function in patients undergoing transplantation for nonalcoholic steatohepatitis cirrhosis: time to reconsider immunosuppression regimens? Liver Transpl. 2011;17:1292-1298.

71. Madhwal S, Atreja A, Albeldawdi M, Lopez R, Post A, Costa MA. Is liver transplantation a risk factor for cardiovascular disease? A meta-analysis of observational studies. Liver Transpl. 2012;18(10):1140-1146.

72. Kennedy C, Redden D, Gray S, et al. Equivalent survival following liver transplantation in patients with non-alcoholic steatohepatitis compared with patients with other liver diseases. HPB (Oxford). 2012;14(9):625-634.

73. Reddy SK, Steel JL, Chen HW, et al. Outcomes of curative treatment for hepatocellular cancer in nonalcoholic steatohepatitis versus hepatitis $\mathrm{C}$ and alcoholic liver disease. Hepatology. 2012;55(6):1809-1819.

74. Hernandez-Alejandro R, Croome KP, Drage M, et al. A comparison of survival and pathologic features of non-alcoholic steatohepatitis and hepatitis $\mathrm{C}$ virus patients with hepatocellular carcinoma. World $J$ Gastroenterol. 2012;18(31):4145-4149.

75. El Atrache MM, Abouljoud MS, Divine G, et al. Recurrence of nonalcoholic steatohepatitis and cryptogenic cirrhosis following orthotopic liver transplantation in the context of the metabolic syndrome. Clin Transplant. 2012;26(5):505-512.

76. Patil DT, Yerian LM. Evolution of nonalcoholic fatty liver disease recurrence after liver transplantation. Liver Transpl. 2012;18(10):1147-1153.

77. Maeda H, Okabayashi T, Nishimori I, Yamashita K, Sugimoto T, Hanazaki K. Hyperglycemia during hepatic resection: continuous monitoring of blood glucose concentration. Am J Surg. 2010;199(1):8-13.
78. Okabayashi T, Ichikawa K, Namikawa T, Sugimoto T, Kobayashi M, Hanazaki K. Effect of perioperative intensive insulin therapy for liver dysfunction after hepatic resection. World J Surg. 2011;35(12): 2773-2778.

79. Marsman HA, de Graaf W, Heger M, et al. Hepatic regeneration and functional recovery following partial liver resection in an experimental model of hepatic steatosis treated with omega-3 fatty acids. Br J Surg. 2013;100(5):674-683.

80. Torres DM, Harrison SA. Diagnosis and therapy of nonalcoholic steatohepatitis. Gastroenterology. 2008;134(6):1682-1698.

81. Belfort R, Harrison SA, Brown K, et al. A placebo-controlled trial of pioglitazone in subjects with nonalcoholic steatohepatitis. $N$ Engl J Med. 2006;355(22):2297-2307.

82. Lavine JE, Schwimmer JB, Van Natta ML, et al; Nonalcoholic Steatohepatitis Clinical Research Network. Effect of vitamin E or metformin for treatment of nonalcoholic fatty liver disease in children and adolescents: the TONIC randomized controlled trial. JAMA. 2011;305(16):1659-1668.

83. Sanyal AJ, Chalasani N, Kowdley KV, et al. Pioglitazone, vitamin E, or placebo for nonalcoholic steatohepatitis. N Engl J Med. 2010;362(18): 1675-1685.

84. Hassan MM, Curley SA, Li D, et al. Association of diabetes duration and diabetes treatment with the risk of hepatocellular carcinoma. Cancer. 2010;116(8):1938-1946.

85. Donadon V, Balbi M, Mas MD, Casarin P, Zanette G. Metformin and reduced risk of hepatocellular carcinoma in diabetic patients with chronic liver disease. Liver Int. 2010;30(5):750-758.

86. Rocha GZ, Dias MM, Ropelle ER, et al. Metformin amplifies chemotherapy-induced AMPK activation and antitumoral growth. Clin Cancer Res. 2011;17(12):3993-4005.

87. Pernicova I, Korbonits M. Metformin - mode of action and clinical implications for diabetes and cancer. Nat Rev Endocrinol. 2014;10(3):143-156.

88. Baselga J, Campone M, Piccart M, et al. Everolimus in postmenopausal hormone-receptor-positive advanced breast cancer. $N$ Engl J Med. 2012;366(6):520-529.

89. Hainsworth JD, Spigel DR, Burris HA 3rd, Waterhouse D, Clark BL, Whorf R. Phase II trial of bevacizumab and everolimus in patients with advanced renal cell carcinoma. J Clin Oncol. 2010;28(13): 2131-2136.

90. Yao JC, Shah MH, Ito T, et al; RAD001 in Advanced Neuroendocrine Tumors, Third Trial (RADIANT-3) Study Group. Everolimus for advanced pancreatic neuroendocrine tumors. $N$ Engl J Med. 2011;364(6):514-523.

91. Tajima K, Nakamura A, Shirakawa J, et al. Metformin prevents liver tumorigenesis induced by high-fat diet in C57B1/6 mice. Am J Physiol Endocrinol Metab. 2013;305(8):E987-E998.

92. Chen HP, Shieh JJ, Chang CC, et al. Metformin decreases hepatocellular carcinoma risk in a dose-dependent manner: population-based and in vitro studies. Gut. 2013;62(4):606-615.

93. Saito T, Chiba T, Yuki K, et al. Metformin, a diabetes drug, eliminates tumor-initiating hepatocellular carcinoma cells. PLoS One. 2013;8(7):e70010.

94. Cai X, Hu X, Cai B, et al. Metformin suppresses hepatocellular carcinoma cell growth through induction of cell cycle G1/G0 phase arrest and p21CIP and p27KIP expression and downregulation of cyclin D1 in vitro and in vivo. Oncol Rep. 2013;30(5):2449-2457.

95. Miyoshi H, Kato K, Iwama H, et al. Effect of the anti-diabetic drug metformin in hepatocellular carcinoma in vitro and in vivo. Int J Oncol. Epub December 30, 2013. 
Journal of Hepatocellular Carcinoma

Dovepress

\section{Publish your work in this journal}

The Journal of Hepatocellular Carcinoma is an international, peerreviewed, open access journal that offers a platform for the dissemination and study of clinical, translational and basic research findings in this rapidly developing field. Development in areas including, but not limited to, epidemiology, vaccination, hepatitis therapy, pathology and molecular tumor classification and prognostication are all considered for publication. The manuscript management system is completely online and includes a very quick and fair peer-review system, which is all easy to use. Visit http://www.dovepress.com/testimonialsphp to read real quotes from published authors.

\footnotetext{
Submit your manuscript here: http://www.dovepress.com/journal-of-hepatocellular-carcinoma-journal
} 